Cahiers
de la Recherche
sur les Droits

Cahiers de la recherche sur les droits

Fondamentaux fondamentaux

$18 \mid 2020$

La vulnérabilité

\title{
La vulnérabilité des usagers des services publics et des consommateurs
}

Delphine Bazin-Beust et Jean-Jacques Thouroude

\section{(2) OpenEdition}

Édition électronique

URL : https://journals.openedition.org/crdf/6442

DOI : $10.4000 /$ crdf.6442

ISSN : 2264-1246

Éditeur

Presses universitaires de Caen

Édition imprimée

Date de publication : 19 novembre 2020

Pagination : 73-81

ISBN : 978-2-84133-987-7

ISSN : $1634-8842$

Référence électronique

Delphine Bazin-Beust et Jean-Jacques Thouroude, «La vulnérabilité des usagers des services publics et des consommateurs ", Cahiers de la recherche sur les droits fondamentaux [En ligne], 18| 2020, mis en ligne le 19 novembre 2021, consulté le 16 novembre 2022. URL : http://journals.openedition.org/ crdf/6442 ; DOl : https://doi.org/10.4000/crdf.6442 


\title{
La vulnérabilité des usagers des services publics et des consommateurs
}

\author{
Delphine BAZIN-BEUST \\ Maître de conférences (HDR) en droit privé à l'université de Caen Normandie \\ Institut Demolombe (EA 967) \\ Jean-Jacques THOUROUDE \\ Maître de conférences émérite en droit public à l'université de Caen Normandie
}

I. La vulnérabilité du consommateur et de l'usager consommateur
A. Le droit de la consommation, réponse à la vulnérabilité du consommateur dans des relations déséquilibrées
1. Le consommateur présumé vulnérable
2. Le consommateur effectivement vulnérable

B. La qualité d'usager d'un service public, risque de neutralisation du droit de la consommation

1. La distinction service public industriel et commercial/service public administratif

2. L'incidence d'une relation non contractuelle ou non exclusivement contractuelle

II. La vulnérabilité des usagers d'un service public administratif

A. La vulnérabilité de l'usager du service public administratif au regard du maintien du service et de ses conditions de fonctionnement

1. L'absence de droit au maintien du service public

2. L'absence de droit au maintien des conditions de fonctionnement du service public

B. Une neutralisation de régimes juridiques favorables

1. Le caractère non écrit d'un contrat passé avec un service public administratif

2. L'impossible bénéfice du régime de la collaboration occasionnelle au service

\begin{abstract}
Si le consommateur est vulnérable, ce n'est pas au titre d'une incapacité d'exercice comme un majeur protégé, mais parce qu'il est un contractant fragilisé ou supposé l'être. L'apparition du droit de la consommation est liée à la société de consommation, loin de l'époque où fut rédigé le Code civil, qui ne partageait pas cette préoccu-
\end{abstract}

pation de protection du particulier face au professionnel par des règles impératives. Sous l'influence du droit de l'Union européenne, cet objectif est rattrapé par celui de la régulation du marché: le consommateur a des droits en tant qu'opérateur intervenant sur un marché dans lequel il doit avoir confiance ${ }^{1}$. Mais pour que ses intérêts

1. P. Stoffel-Munck, «L'autonomie du droit contractuel de la consommation: d'une logique civiliste à une logique de régulation», Revue trimestrielle de droit commercial, 2012, p. 705. 
soient préservés, il doit pouvoir faire des choix éclairés et accéder aux biens et services; ce qui n'est pas toujours le cas des consommateurs précarisés socialement et/ou économiquement. Par ailleurs, face au service public, l'usager se présente comme un consommateur à géométrie variable, exposé à une vulnérabilité juridique. De fait, au gré de la nature de la relation entretenue avec ce service, il est ou non privé des règles consuméristes attachées à sa qualité de partie à un contrat de consommation conclu avec un professionnel (I).

La vulnérabilité juridique des usagers des services publics administratifs et, dans une moindre mesure, des usagers des services publics industriels et commerciaux dont le juge administratif n'a à connaître qu'en ce qui concerne les recours contre les mesures générales d'organisation de ces services ou la suppression du service, tient à la situation dite «légale et réglementaire» dans laquelle ils se trouvent à l'égard du service. Ils se trouvent en situation de précarité juridique, qu'il s'agisse de la création des services publics administratifs, à laquelle ils n'ont aucun droit, sauf pour les services publics obligatoires, ou, lorsqu'ils accèdent à la qualité d'usager du service public administratif, du fonctionnement du service ou de sa suppression (II)

\section{La vulnérabilité du consommateur et de l'usager consommateur}

Traditionnellement, les opérateurs gérant des services publics ne relèvent pas des règles consuméristes, faute pour leurs usagers d'être des clients. Or certains d'entre eux tiennent un discours commercial, développent des pratiques promotionnelles et incitatives comme le font des entreprises privées. Si le droit de la consommation s'est construit pour remédier au déséquilibre quasi inhérent de la relation consommateur/professionnel (A), son application à l'usager d'un service public appelle une réponse nuancée (B).

\section{A. Le droit de la consommation, réponse à la vulnérabilité du consommateur dans des relations déséquilibrées}

Dans l'esprit originaire du droit de la consommation, la vulnérabilité du consommateur est davantage présumée (1) que constatée (2).

\section{Le consommateur présumé vulnérable}

Rien dans la définition légale du consommateur ne renvoie à sa fragilité: «[...] toute personne physique qui agit à des fins qui n'entrent pas dans le cadre de son activité commerciale, industrielle, artisanale, libérale ou agricole $»^{2}$. Si la qualification de majeur protégé repose sur une analyse individuelle, celle de consommateur dépend de la finalité extraprofessionnelle de l'action, sans considération d'une faiblesse réelle. L'adepte de nouvelles technologies achetant un ordinateur ou l'investisseur effectuant du trading en ligne sur le marché des devises FOREX ne peut donc se voir dénier cette qualité ${ }^{3}$. Cette approche objective et abstraite s'avère adaptée à un droit de masse et à un contentieux de masse.

Mais le droit de la consommation repose sur un double postulat: le consommateur est potentiellement vulnérable face au professionnel et celui-ci est potentiellement susceptible d'abuser de sa supériorité. C'est sur un rapport de force inégal, dont la vulnérabilité contractuelle est sous-jacente, présumée, que repose ce droit social du marché ${ }^{4}$ qui ne s'applique pas en principe en $B$ to $B$, même si le professionnel contracte en dehors de son domaine de compétence, ni en $C$ to $C$. Certes, tous les consommateurs ne sont pas démunis et ne se contentent plus de consommer, offrant des biens et services sur le marché. D'où le refus de qualifier de consommateur celui qui se déclare loueur professionnel de logements financés à crédit pour profiter d'une défiscalisation 5 .

Des procédés consuméristes emblématiques sont particulièrement adaptés à des vulnérabilités contractuelles émergentes. Le juge n'hésite pas à utiliser le droit de la consommation pour préserver les intérêts des consommateurs dans une société qui se digitalise, sans pour autant rechercher leur protection inconditionnelle et imposer des obligations disproportionnées aux professionnels ${ }^{6}$.

Dans le commerce électronique, l'information précontractuelle du consommateur est un remède à l'éloignement des parties. Toutefois une plateforme, type Amazon, n'a pas à communiquer, avant la conclusion du contrat, un numéro de téléphone pour la joindre s'il existe un moyen de communication alternatif équivalent ${ }^{7}$. L'information précontractuelle sert aussi à imposer de la transparence et de la loyauté aux plateformes ${ }^{8}$. L'utilisateur d'une place de marché en ligne ou d'un site comparateur est informé du référencement payant des offres et de la qualité de l'opérateur avec lequel il est mis en relation, professionnel ou consommateur'.

2. Article liminaire du Code de la consommation.

3. Cass., $1^{\text {re }}$ civ., 22 janvier 2014, $\mathrm{n}^{\circ}$ 12-20982; CJUE, 3 octobre 2019, C-208/18.

4. J.-P. Chazal, «Vulnérabilité et droit de la consommation», communication au colloque sur la vulnérabilité et le droit, université de Grenoble, 23 mars 2000, en ligne: https://hal-sciencespo.archives-ouvertes.fr/hal-01053489/document.

5. Cass., $1^{\text {re }}$ civ., 23 janvier 2019, $\mathrm{n}^{\circ} 17-23917$.

6. D. Bazin-Beust, «Digitalisation et consommation», Revue de jurisprudence commerciale, mai-juin 2019, p. 198.

7. CJUE, 10 juillet 2019, C-649/17; directive (UE) 2019/2161 du Parlement européen et du Conseil du 27 novembre 2019 dite «Omnibus » à transposer au 28 novembre 2021, art. 4 .

8. Art. L. 111-7 du Code de la consommation, issu de la loi $n^{\circ}$ 2016-1321 du 7 octobre 2016.

9. Directive (UE) 2019/2161, art. 6 bis. 
Le droit des clauses abusives est mobilisé pour protéger le consommateur contractant avec les réseaux sociaux et plateformes digitales, à la lisière du droit de la protection des données personnelles ${ }^{10}$. Ont été sanctionnées, souvent sur action des associations de consommateurs, les clauses suivantes: celle de Twitter prétendant que les contrats sont conclus à titre gratuit en l'absence de contrepartie monétaire due par l'utilisateur ${ }^{11}$; de Facebook imposant de saisir les tribunaux californiens ${ }^{12}$; d'Amazon faisant croire que seul le droit luxembourgeois s'applique sans préciser que le consommateur relève du droit de la consommation du pays de sa résidence ${ }^{13}$.

Pour le droit de rétractation de quatorze jours lié aux contrats à distance ou hors établissement, c'est un facteur aggravant de vulnérabilité qui déclenche la protection du consommateur: un lieu non commercial favorise l'exploitation de sa fragilité. Des professionnels en ligne tentent d'y faire échec mais le juge a une interprétation restrictive des exclusions légales. La plaque d'immatriculation d'une moto n'en fait pas un bien «nettement personnalisé » ${ }^{14}$. Un matelas déballé peut être restitué à l'inverse d'une marchandise qui, pour des raisons d'hygiène, ne pourrait l'être ${ }^{15}$.

Le droit des pratiques commerciales déloyales sanctionne des méthodes exploitant la crédulité et la captivité des consommateurs, confinant à des arnaques. En janvier 2020, l'UFC-Que choisir a assigné le site LastMinute ${ }^{16}$ pour pratiques trompeuses et agressives: en cliquant sur une fenêtre $p o p$ - $u p$ après renseignement de sa carte bancaire, le client souscrit une assurance voyages! La vente en ligne de grilles de jeux Loto et Euromillions a été condamnée comme pratique présumée trompeuse en tant qu'elle affirme augmenter la chance de gagner aux jeux de hasard ${ }^{17}$. Si la vente d'ordinateurs pré-équipés de logiciels n'est pas per se une pratique déloyale car elle correspond à la demande de la majorité de la clientèle ${ }^{18}$, celle de cartes SIM comprenant des services payants pré-installés et activés dont le consommateur n'est pas informé est une pratique agressive en tant que fourniture non demandée ${ }^{19}$.
Le juge démontre qu'il n'applique pas aveuglément les remèdes consuméristes fondés sur une vulnérabilité contractuelle présumée, distincte de la vulnérabilité avérée liée à une faiblesse subjective du consommateur.

\section{Le consommateur effectivement vulnérable}

Il est parfois tenu compte de la situation concrète de fragilité des consommateurs en raison de leur âge, état de santé, déficience intellectuelle pour en déduire un régime juridique spécifique. C’est le cas des «séniors». Si tous ne souffrent pas de déficit auditif, visuel ou cognitif, le vieillissement est propice à la vulnérabilité. Il a été proposé de porter à trente jours le délai de rétractation en cas de démarchage à domicile de clients de plus de $70 \mathrm{ans}^{20}$. Depuis la loi no 2014-366 du 24 mars 2014 pour l'accès au logement et un urbanisme rénové (loi ALUR), le bailleur - professionnel ou non - d'un locataire de plus de 65 ans aux ressources modestes ne peut donner congé que s'il lui propose un logement de remplacement «correspondant à ses besoins et à ses possibilités " situé dans une zone proche du logement occupé ${ }^{21}$.

La vulnérabilité subjective du consommateur permet surtout de moduler l'appréciation de la déloyauté et de l'agressivité d'une pratique déployée par un professionnel. Conformément au droit européen, les pratiques commerciales déloyales doivent être analysées in concreto quand elles visent une " catégorie particulière de consommateurs ou un groupe de consommateurs vulnérables en raison d'une infirmité mentale ou physique, de leur âge ou de leur crédulité », et non plus à l'aune du consommateur «normalement informé et raisonnablement attentif et avisé $»^{22}$. Pour déterminer si la pratique est agressive, on doit prendre en compte

[...] l'exploitation, en connaissance de cause, par le professionnel, de tout malheur ou circonstance particulière d'une gravité propre à altérer le jugement du consommateur ${ }^{23}$.

10. J. Sénéchal, «Lutte contre les clauses abusives et protection des données à caractère personnel», Actualité juridique. Contrat, 2019, p. 412. La loi $\mathrm{n}^{\circ}$ 2016-1321 du 7 octobre 2016 accordait au consommateur un droit à la portabilité de ses données (art. L. 224-42-1 du Code de la consommation). Il fut supprimé par la loi no 2018-493 du 20 juin 2018, le RGPD (Règlement général sur la protection des données - règlement (UE) 2016/679 du Parlement européen et du Conseil du 27 avril 2016) entré en vigueur le 25 mai 2018 instaurant ce droit pour toute personne physique (art. 20).

11. TGI Paris, 7 août $2018, \mathrm{n}^{\circ} 14 / 07300$

12. CA Paris, 12 février $2016, \mathrm{n}^{\circ} 15 / 08624$.

13. CJUE, 28 juillet 2016, C-191/15.

14. Cass., $1^{\text {re }}$ civ., 20 mars $2013, \mathrm{n}^{\circ} 12-15.052$

15. CJUE, 27 mars 2019, C-681-17.

16. Voir «LastMinute - Consommateurs, ne vous laissez pas abuser», 27 janvier 2020, en ligne: https://www.quechoisir.org/action-ufc-que-choisirlastminute-consommateurs-ne-vous-laissez-pas-abuser-n74807.

17. Cass. crim., 28 janvier $2020, \mathrm{n}^{\circ} 19-80496$

18. Cass., $1^{\text {re }}$ civ., 29 mars $2017, n^{\circ} 15-13248$.

19. CJUE, 13 septembre 2018, C-54/17.

20. Assemblée nationale, proposition de loi $n^{\circ} 3633$ visant à renforcer la protection des personnes âgées à l'égard du démarchage, enregistrée à la présidence de l'Assemblée nationale le 30 janvier 2007, antérieure à la directive 2011/83/UE du Parlement européen et du Conseil du 25 octobre 2011 réformant les contrats hors établissement et fixant le délai de rétractation à quatorze jours. La directive 2019-2161, cons. 54, permet d'étendre ce délai pour les ventes conclues lors de visites à domicile car elles ciblent des personnes âgées ou d'autres consommateurs vulnérables.

21. Art. 15, III de la loi no 89-462 du 6 juillet 1989; le dispositif est écarté si le bailleur présente les mêmes conditions de vulnérabilité d'âge et de ressources.

22. Art. L. 121-1, al. 3 du Code de la consommation; directive 2005-29/CE du Parlement européen et du Conseil du 11 mai 2005 , art. 5.

23. Art. L. 121-6, al. 2, $3^{\circ}$ du Code de la consommation. 
Le droit de l'Union européenne inclut aussi parmi ses objectifs la satisfaction des besoins spécifiques des consommateurs vulnérables ${ }^{24}$. Les causes de vulnérabilité des secteurs "particulièrement problématiques » visés sont endogènes (âge, handicap) et exogènes (méconnaissance de la langue, manque d'informations, nouvelles technologies, etc. ${ }^{25}$. Sont concernés notamment des secteurs économiques ouverts à la concurrence, anciennement sous monopole public, et générateurs de nouvelles précarités: fracture numérique, technologique, énergétique ${ }^{26}$. De fait, certains consommateurs ne peuvent profiter des bienfaits du marché unique en raison de leur impossibilité d'utiliser le commerce électronique ou l'intelligence artificielle pour gérer leur consommation énergétique ou produire de l'énergie, les privant de prix concurrentiels et les exposant à des coûts plus élevés pour ces dépenses contraintes.

Le droit français s'inscrit dans cette voie. Il impose aux entreprises dont le chiffre d'affaires est supérieur à 250 millions d'euros de rendre leur numéro de téléphone pour traiter une réclamation accessible gratuitement aux personnes «sourdes, malentendantes, aveugles et aphasiques » via un service de traduction simultanée écrite et visuelle ${ }^{27}$. Les fournisseurs d'électricité et de gaz naturel doivent adapter la communication de leurs contrats et informations aux handicaps des consommateurs ${ }^{28}$. En janvier 2019, l'UFC-Que choisir a saisi le Conseil d'État pour le retard à l'adoption des décrets sur l'affichage en euros des compteurs Linky pour les bénéficiaires du chèque énergie prévu par la loi du 17 août 2015 relative à la transition énergétique ${ }^{29}$.

Le consommateur vulnérable est un standard complétant celui du consommateur moyen, trop rigide face aux fragilités pouvant surgir au cours de la vie. Mais il n'est pas défini uniformément par le droit de l'Union européenne et ses préconisations sont variables. Soit garantir l'accès à des biens et services en imposant aux professionnels une adaptation de leur infrastructure ou des tarifs encadrés, telle une lutte contre l'exclusion sociale significative d'un «para-droit de la consommation ». Soit moduler l'appréciation d'une pratique commerciale en mettant l'accent sur un risque d'exploitation de la fragilité contextualisée du consommateur. C'est alors un correctif à l'approche objective du consommateur, à l'application mécanique d'un droit de la consommation jugé parfois trop libéral et sous obédience du marché. Si la vulnérabilité subjective n'aboutit pas en tant que telle à consacrer des droits nouveaux, sa généralisation risque de faire éclore un droit de la consommation à deux vitesses, du moins d'amplifier l'approche catégorielle de ces bénéficiaires. Voyons ce qu'il en est de l'usager d'un service public invoquant le droit de la consommation.

\section{B. La qualité d'usager d'un service public, risque de neutralisation du droit de la consommation}

La soumission du prestataire de service public au droit de la consommation dépend en premier lieu de l'activité qu'il déploie, marchande ou non (1). Quant aux droits de l'usager consommateur, ils sont tributaires d'une relation contractuelle conclue avec le professionnel déployant ce service public (2). La nature des liens noués avec le prestataire conditionnant l'application des textes consuméristes, l'usager consommateur se trouve exposé à une vulnérabilité d'ordre juridique.

\section{La distinction service public industriel et commercial/service public administratif}

L'usager ne cumule la qualité de consommateur que s'il traite avec un service comparable à une entreprise intervenant sur un marché. L'usager d'un service public industriel et commercial a pour partenaire un professionnel, personne morale publique ou privée agissant à des fins entrant dans le cadre de son activité commerciale, industrielle, artisanale, libérale ou agricole ${ }^{30}$. À la différence d'un service public administratif ne déployant pas d'activité économique pour une clientèle contre rémunération, le service public industriel et commercial va basculer dans le droit de la consommation ${ }^{31}$. Est un professionnel l'établissement à but non lucratif poursuivant une mission d'intérêt général d'enseignement et accordant, à titre accessoire, une facilité de paiement à

24. Résolution du Parlement européen concernant une stratégie de renforcement des droits des consommateurs vulnérables, 22 mai 2012, 2011/2272(INI); M. Friant-Perrot, «Le consommateur vulnérable à la lumière du droit de la consommation de l’Union européenne», Revue trimestrielle de droit européen, vol. 49, $\mathrm{n}^{\circ} 3,2013$, p. 483-498.

25. Alimentation, transports, Internet, énergie, accès à la justice.

26. Résolution du Parlement européen sur la protection des consommateurs dans les services collectifs : énergie, télécommunications, services postaux, transports publics, 15 avril 2014, 2013/2153(INI); résolution du Parlement européen sur une nouvelle donne pour les consommateurs d'énergie, 26 mai 2016, 2015/2323(INI).

27. Art. L. 112-8 du Code de la consommation.

28. Art. L. 224-2 du Code de la consommation.

29. Voir «Linky - Afficheur déporté: l’UFC-Que choisir saisit le Conseil d'État», 29 janvier 2019, en ligne: https://www.quechoisir.org/action-ufcque-choisir-linky-afficheur-deporte-l-ufc-que-choisir-saisit-le-conseil-d-etat-n63223.

30. Article liminaire du Code de la consommation.

31. F. Béroujon, "Application du droit de la consommation à un service public administratif: refus de principe ou excès de prudence du juge administratif?», Les petites affiches, n $177-178,5$ septembre 2012, p. 20. En octobre 2019, Engie a été condamné pour démarchage illégal. Selon la Direction générale de la concurrence, de la consommation et de la répression des fraudes (DGCCRF), le consommateur démarché à domicile ou par téléphone est plus vulnérable qu'en établissement commercial (DGCCRF, «La DGCCRF sanctionne Engie pour démarchage abusif», communiqué de presse, 18 octobre 2019, en ligne, https://www.economie.gouv.fr/files/files/directions_services/dgccrf/presse/communique/2019/ CP-dgccrf-sanctionne-engie-pour-demarchage-abusif.pdf). 
un élève ${ }^{32}$; de même de l'établissement public hospitalier ou de maison de retraite pour les activités autres que de soins (restauration, location de biens, hébergement) ${ }^{33}$. Pour la Commission européenne, la «quasi-totalité» des services sociaux - dont le logement social - devrait être considérée comme des activités économiques au sens du traité instituant la Communauté européenne, soit des prestations fournies normalement contre rémunération même non payées directement par les bénéficiaires ${ }^{34}$.

L'assimilation du service public industriel et commercial au professionnel fut par le passé préconisée par la Commission des clauses abusives dans les contrats de distribution d'eau ${ }^{35}$. Elle a surtout été consacrée par le Conseil d'État qui applique la législation sur les clauses abusives entre les délégataires de service public et leurs usagers ${ }^{36}$, lesquels, ici d'ailleurs, sont des sociétés commerciales inqualifiables de non professionnelles ${ }^{37}$ bénéficiaires de l'article L. 212-1 du Code de la consommation sur les clauses abusives. En déclarant illégale la disposition mettant à la charge des usagers les dommages liés à une fuite d'eau, soit une clause exonératoire de responsabilité, le juge administratif contrôle l'application du droit de la consommation, comme son homologue judiciaire, et intègre la législation consumériste sur les clauses abusives dans le bloc de légalité de l'administration.

Mais la particularité de la relation service public industriel et commercial / usager peut modifier, voire exclure, la protection du Code de la consommation et générer une vulnérabilité d'ordre juridique à l'égal de l'usager d'un service public administratif.

\section{L'incidence d'une relation non contractuelle ou non exclusivement contractuelle}

La directive 93/13 sur les clauses abusives exclut les «clauses [contractuelles] qui reflètent des dispositions législatives ou réglementaires impératives ${ }^{38}$. D’harmonisation minimale, le droit français n'a pas transcrit cette exclusion. Ce qui a permis au Conseil d'État de contrôler l'abus d'un règlement de distribution d'eau dont les dispositions, insérées dans un contrat d'adhésion, sont opposées à l'abonné. L'appréciation du déséquilibre significatif déborde alors sur des dispositions qui n'ont pas fait l'objet d'un accord entre les parties.

Or la nature du lien avec l'usager aurait pu constituer un obstacle au droit de la consommation, conditionné à l'existence d'une relation contractuelle. Des services publics agissent par décisions unilatérales. Des gestionnaires invoquent comme ici contre l'usager des clauses «reflétant» des dispositions légales ou réglementaires qui régissent le fonctionnement du service et leur sont imposées par cahier des charges. Le juge administratif ne s'y est pas arrêté. Toutefois son application du droit consumériste n'est pas sans limite puisqu'il précise que le caractère abusif d'une clause s'apprécie au regard tant de la clause et de l'ensemble des stipulations du contrat qu'au regard «des caractéristiques particulières» du service quand le contrat a pour objet l'exécution d'un service public. La demande de l'usager en réputé non écrit sera alors rejetée si la clause - exorbitante du droit commun - est justifiée par les nécessités du service, l'intérêt général. Le standard d'appréciation de l'abus est là encore considérablement modifié par rapport au Code de la consommation.

La spécificité de la relation service public industriel et commercial / usager peut aussi motiver l'exclusion d'une règle procédurale consumériste. Un particulier opposait à la collectivité publique émettant un titre exécutoire contre lui la prescription de son action en paiement de la redevance. Il est débouté car l'usager d'un service public de l'enlèvement des ordures ménagères n'étant pas lié à ce service par un contrat, le délai n'est pas soumis aux dispositions dérogatoires de l'article L. 137-2, devenu L. 218-2 du Code de la consommation ${ }^{39}$. La personne publique professionnelle échappe à la prescription biennale au profit d'une prescription quadriennale.

La Cour de cassation ne dit rien sur la nature de la relation, probablement légale puisque la redevance a été prise en application de l'article L. 23333-76 du Code général des collectivités territoriales. Mais, en se cristallisant sur l'absence de relation contractuelle, ne marque-t-elle pas un recul par rapport au juge administratif? Sauf à dire que là, il n'y a même pas de clause reflétant une disposition légale puisque la redevance résulte d'un acte unilatéral de la collectivité. Elle persiste aussi à réserver la prescription biennale au consommateur lié par contrat à un professionnel ${ }^{40}$. Or la lettre de l'article L. 218-2 du Code de la consommation ne l'exige pas - même si son insertion dans le livre II "Formation et exécution des contrats» du Code milite en ce sens - et une réponse ministérielle préconisait ce délai biennal pour les redevances d'eau et d'assainissement ${ }^{41}$. Cette restriction ne devrait pas jouer pour un service public poursuivi pour pratique commerciale déloyale, laquelle est toute action, omission, etc., d'un professionnel «en relation directe

32. CJUE, 17 mai 2018, C-147/16.

33. La directive 2013/11/UE du Parlement européen et du Conseil du 21 mai 2013 sur la médiation consommation exclut les services d'intérêt général non économiques et les services de santé (art. 2, 2, c et h).

34. Commission européenne, $\operatorname{Com}(2006) 177$ final, 26 avril 2006.

35. Commission des clauses abusives, recommandations $n^{\circ} 85-01,19$ novembre 1982 et $n^{\circ}$ 01-01, 25 janvier 2001.

36. CE, 11 juillet 2001, Société des eaux du Nord, $\mathrm{n}^{\circ}$ 221458; CE, 30 décembre 2015, Compagnie méditerranéenne des cafés Malengo, $\mathrm{n}^{\circ} 387666$.

37. «[...] toute personne morale qui n'agit pas à des fins professionnelles» (article liminaire du Code de la consommation).

38. Directive 93/13/CEE du Conseil du 5 avril 1993, cons. 13.

39. Cass., $1^{\text {re }}$ civ., 4 juillet 2019, $n^{\circ} 19-13.494$

40. Cass., $1^{\text {re }}$ civ., 9 juin $2017, \mathrm{n}^{\circ} 16-21.247$, pour un refus à une gestion d'affaires.

41. Réponse du ministère des Finances et des Comptes publics à la question écrite n 12641 du sénateur P. Leroy, 25 mai 2016 , en ligne: https://www. senat.fr/questions/base/2014/qSEQ140712641.html. 
avec la promotion, la vente ou la fourniture d'un produit aux consommateurs ${ }^{42}$

L'étude de l'usager-consommateur nous rappelle que le droit de la consommation est avant tout un droit des contrats de consommation, des relations contractuelles inégalitaires ou présumées comme telles, tributaires de rapports de force économiques et issues d'un acte unilatéralement élaboré par le professionnel mais donnant lieu à accord de volontés ${ }^{43}$. Quant à la définition finaliste du consommateur - personne physique agissant à titre extraprofessionnel -, elle révèle, à l'heure où il utilise des plateformes en ligne pour proposer des biens et services, qu'il est aussi un acteur économique sur le marché. Reste alors à définir les contours de la non-professionnalité mais c'est une autre question...

L'usager d'un service public administratif est pour sa part totalement éloigné de la notion de consommateur et sa vulnérabilité est davantage juridique qu'économique.

\section{La vulnérabilité des usagers d'un service public administratif}

Les usagers des services publics à caractère administratif sont principalement les victimes de l'une des fameuses lois du service public, dites «lois de Rolland» (1938), à savoir la loi de mutabilité ou d'adaptation du service même si la loi d'égalité a le mérite de favoriser un accès «égal » au service public ${ }^{44}$.

Les usagers du service public n'ont ainsi aucun droit au maintien du service public ou de ses conditions de fonctionnement (A) et ne peuvent par ailleurs bénéficier de régimes juridiques neutralisés par le juge alors qu'ils pourraient pourtant atténuer leur vulnérabilité juridique (B).

\section{A. La vulnérabilité de l'usager du service public administratif au regard du maintien du service et de ses conditions de fonctionnement}

\section{L'absence de droit au maintien du service public}

Alors même qu'un service public a été régulièrement créé par une personne publique, les usagers de ce service ne peuvent faire valoir aucun droit acquis à son maintien.

Il en a été ainsi jugé par exemple pour la fermeture d'une clinique ouverte dans un arrêt du Conseil d'État du 6 avril 1973. Dans cette décision les praticiens exerçant dans une clinique ouverte contestaient la décision prise par la commission administrative de cet établissement de fermer cette clinique en se prévalant des droits qu'ils auraient acquis au maintien de cette clinique. Le Conseil d'État rejette ce moyen en énonçant le principe selon lequel, sous réserve de respecter les règles qui s'imposent à elle, il appartient à l'autorité administrative de décider la suppression d'un service public «sans que les usagers de ce dernier puissent utilement invoquer un droit acquis à son maintien ${ }^{45}$.

La même solution a été retenue dans un arrêt du Conseil d'État du 25 avril $1994^{46}$ à propos de la suppression d'une classe unique dans un hameau à raison d'un effectif d'élèves insuffisant. Aux termes de l'article 11 de la loi du 30 octobre 1886 sur l'organisation de l'enseignement primaire, alors applicable et abrogée depuis le 22 juin 2000,

Toute commune doit être pourvue au moins d'une école primaire publique. Il en est de même de tout hameau séparé du chef-lieu ou de toute autre agglomération par une distance de trois kilomètres et réunissant au moins quinze enfants d'âge scolaire.

En l'espèce une classe d'un hameau de montagne avait été supprimée par l'inspecteur d'académie, l'emploi d'instituteur de cette classe ayant été rattaché à la commune principale bénéficiant d'une école primaire relevant du service public obligatoire. Cette suppression de classe a été jugée légale par le Conseil d'État au regard du nombre d'élèves la fréquentant (sept), inférieur à quinze, et en application du principe selon lequel

[...] les usagers d'un service public qui n'est pas obligatoire n'ont aucun droit au maintien de ce service au fonctionnement duquel l'administration peut mettre fin lorsqu'elle l'estime nécessaire.

\section{L'absence de droit au maintien des conditions de fonctionnement du service public}

La vulnérabilité juridique des usagers du service public administratif résulte en la matière de leur situation dite légale et réglementaire. Les droits qu'ils peuvent tirer de ces textes ne valent que tant que ces textes ne sont pas abrogés ou modifiés. L'illustration de cette précarité statutaire se retrouve aussi bien en matière de délivrance de diplômes universitaires (a) que de fixation des tarifs d'accès aux services publics (b).

\section{a. L'absence de pérennité de la délivrance de diplômes universitaires}

Dans un arrêt en date du 12 février 1982, le Conseil d'État a consacré le principe de l'absence de droit des étudiants

42. Directive 2005/29/CE du Parlement européen et du Conseil du 11 mai 2005, art. 2, d.

43. T. Revet, «Le projet de réforme et les contrats structurellement déséquilibrés », Recueil Dalloz, 2015, p. 1217; directive (UE) 2019/2161, art. 4 visant les contrats de fourniture d'eau, gaz, électricité ou chauffage urbain, "par des prestataires publics, dans la mesure où ces produits sont fournis sur une base contractuelle».

44. CE, 5 mars 1951, Société des concerts du conservatoire, $\mathrm{n}^{\circ}$ 92004: illégalité de la décision du directeur de la Radiodiffusion française de diffuser les concerts de cette seule société.

45. CE, 6 avril $1973, \mathrm{n}^{\circ} 83281$

46. CE, 25 avril $1994, \mathrm{n}^{\circ} 137793$. 
au maintien des diplômes délivrés par une université suite à un refus ministériel d'habilitation de ces diplômes à l'université Paris 7, génie biologique et médical, littérature française et comparée, histoire et civilisations. Le considérant de principe de cet arrêt mérite d'être reproduit tant il traduit clairement la situation juridiquement précaire des étudiants usagers du service public universitaire:

Considérant [...] que le statut des usagers du service public de l'enseignement supérieur est défini par des textes législatifs et réglementaires et que le droit aux avantages qui résultent pour eux de ce statut est subordonné au maintien en vigueur des textes qui les leur confèrent; qu'il suit de là que la vocation des étudiants de l'université requérante à poursuivre dans cet établissement des études en vue de l'obtention de certains diplômes nationaux conférant les titres ou grades suivants, à savoir le diplôme d'études approfondies, le doctorat de troisième cycle, le diplôme d'études supérieures spécialisées, le diplôme de docteuringénieur, était subordonné au maintien en vigueur des dispositions réglementaires habilitant cet établissement à délivrer ces diplômes; qu'ainsi l'université requérante n'est pas fondée à invoquer de prétendus droits acquis par ses étudiants en cours d'études pour demander l'annulation des décisions attaquées en tant qu'elles ne prévoient pas de dispositions transitoires au bénéfice desdits étudiants ${ }^{47}$.

\section{b. L'absence de droit acquis au maintien des tarifs existants d'un service public administratif}

La solution a été dégagée par exemple pour l'augmentation des tarifs d'une cantine scolaire décidée par la caisse des écoles dans un arrêt de la cour administrative d'appel de Paris du 2 juillet 2010:

Considérant que si les requérants soutiennent que la majorité des familles subirait une augmentation des tarifs du fait du nouveau dispositif, ils ne sauraient se prévaloir d'aucun droit au maintien des tarifs préexistants, d'une part, et n'établissent pas, ni d'ailleurs n'allèguent, que les tarifs issus du nouveau dispositif seraient supérieurs au coût par usager de la prestation ou qu'ils feraient obstacle à l'égal accès de tous les usagers au service ${ }^{48}$.

L'absence de maintien garanti des tarifs se retrouve également en matière de tarification de l'accès aux musées. Le Conseil d'État s'est prononcé sur cette question dans un arrêt du 18 janvier 2013:

Considérant [...] que les tarifs d'un musée ou d'un monument national, y compris lorsqu'ils comprennent un droit d'accès gratuit pour une partie des usagers, même motivé par la poursuite d'un objectif social, ne constituent pas la traduction d'un droit qui pourrait être regardé comme une créance des usagers sur l'État dont la privation porterait atteinte au droit de propriété tel qu'il est garanti par l'article premier du premier protocole additionnel à la convention européenne de sauvegarde des droits de l'homme et des libertés fondamentales; que, dès lors, le moyen tiré de ce que les limitations apportées par les délibérations attaquées à la gratuité de l'accès aux musées concernés méconnaîtrait ces stipulations, combinées avec celles de l'article 14 de la même convention, doit être écarté ${ }^{49}$.

\section{B. Une neutralisation de régimes juridiques favorables}

La vulnérabilité juridique des usagers des services publics administratifs se trouve aggravée par le constat jurisprudentiel de l'impossibilité pour eux de bénéficier de régimes juridiques aussi protecteurs que le contrat administratif, jugé incompatible avec leur situation «statutaire et réglementaire» (1) ou la qualification de collaborateur occasionnel du service public (2).

\section{Le caractère non écrit d'un contrat passé avec un service public administratif}

Le principe de l'absence de toute situation contractuelle a été à de nombreuses reprises rappelé par le Conseil d'État dans des domaines aussi variés que l'aide sociale (a), l'hospitalisation de patients (b) ou la préparation de thèses (c).

\section{a. L'absence de toute situation contractuelle pour les usagers de l'aide sociale}

Concernant les enfants dans des crèches, le Conseil d'État a jugé le 21 septembre $1990^{50}$ que les crèches gérées par les services de l'action sociale des armées du ministère de la Défense et destinées à recevoir les enfants en bas âge des ressortissants de ce ministère ont le caractère d'un service public administratif. Les usagers de ce service public ne sont pas placés, vis-à-vis de l'État, qui en assure directement la gestion, dans une situation contractuelle.

Pour les usagers du service d'aide à domicile, le Conseil d'État a précisé le 5 juillet 2017, dans une affaire révélant l'existence d'un «vrai-faux» contrat de séjour pour l'aide à domicile, que la prise en charge de prestations d'aide à domicile par un centre communal d'action sociale constitue un service public administratif:

47. CE, 12 février 1982, n 27098, 27099, 27700 (nous soulignons). Participe du même esprit la solution adoptée par le Conseil d'État dans un arrêt du 24 janvier 2001 ( $n^{\mathrm{o}}$ 229501) : l'accès à une formation de $3^{\mathrm{e}}$ cycle de l'enseignement supérieur, en l'espèce un diplôme d'études approfondies (DEA) de droit médical, ne constitue pas une liberté fondamentale dont la sauvegarde est susceptible de donner lieu au prononcé des mesures sur le fondement de l'article L. 521-2 du Code de justice administrative.

48. CAA Paris, 2 juillet 2010, $\mathrm{n}^{\circ}$ o9PAoo974. La même solution vaut pour les usagers des services publics industriels et commerciaux. Voir en ce sens CAA Paris, 24 novembre 2015, n 14 PA01350: il s'agissait en l'espèce des tarifs de la distribution d'eau potable.

49. CE, 18 janvier 2013, $\mathrm{n}^{\circ} 328230$, cons. 8 (nous soulignons). Cette délibération a néanmoins été annulée par le Conseil d’État au motif qu'en limitant aux seules personnes de 18 à 25 ans ressortissant d'un État membre de l'Union européenne ou d'un État partie à l'Espace économique européen la gratuité d'accès aux monuments dont ils ont la charge et en excluant ainsi les résidents de longue durée en situation régulière de ces mêmes États la disposition en cause a méconnu les dispositions de la directive 2003/109/CE du Conseil du 25 novembre 2003.

50. CE, 21 septembre $1990, n^{\circ} 87408$. 
Considérant que [...] les usagers de ce service public ne sauraient être regardés comme placés dans une situation contractuelle vis-à-vis de l'établissement concerné, alors même qu'ils concluent avec celui-ci un "contrat de séjour» ou qu'est élaboré à leur bénéfice un "document individuel de prise en charge», dans les conditions fixées par l'article L. 311-4 du [code de l'action sociale et des familles]; que le moyen tiré de ce qu'un litige opposant un tel service public administratifà un de ses usagers ne peut être réglésur un fondement contractuel est relatif au champ d'application de la loi et est, par suite, d'ordre public ${ }^{51}$.

Le Conseil d'État a enfin jugé dans un arrêt du 4 décembre 2019 que les usagers bénéficiaires du revenu de solidarité active (RSA) sont victimes d'une "double peine» puisqu'ils ne bénéficient pas d'un véritable contrat et que le «contrat», ainsi improprement qualifié, est inattaquable directement devant le juge administratif par les bénéficiaires du RSA qui peuvent seulement se prévaloir de son illégalité éventuelle à l'occasion d'un recours contre une décision de suspension du versement du RSA.

Selon le Conseil d'État,

L'obligation de conclure un «contrat librement débattu», prévue aux articles L. 262-35 et L. 262-36 du code de l'action sociale et des familles, n'a ni pour objet ni pour effet de placer le bénéficiaire du revenu de solidarité active dans une situation contractuelle vis-à-vis du département qui lui verse ce revenu. Il suit de là qu'en regardant le «contrat d'engagement» signé par $\mathrm{M}^{\mathrm{me}} \mathrm{B}$... le 25 novembre 2009 comme un contrat de droit public et en estimant, par suite, qu'il était saisi d'un litige contractuel, le tribunal administratif de Strasbourg a méconnu le champ de la loi.

Toutefois, si le contenu de ce document peut être discuté, le cas échéant, à l'occasion d'un recours formé contre une décision de suspension du versement du revenu de solidarité active prise sur le fondement de l'article L. 262-37 du code de l'action sociale et des familles, ce document n'a pas le caractère d'un acte faisant grief. Par suite, le «contrat d'engagement» signé par $\mathrm{M}^{\text {me }} \mathrm{B}$... le 25 novembre 2009 n'était pas susceptible de recours et les conclusions tendant à son annulation étaient irrecevables. Il y a lieu de substituer ce motif, qui est d'ordre public et n'appelle l'appréciation d'aucune circonstance de fait, aux motifs du jugement attaqué, dont il justifie le dispositif ${ }^{52}$.

Cette requérante aurait dû en l'espèce attaquer la décision suspendant le versement de son RSA et non le contrat lui-même.

\section{b. L'absence de contrat pour les patients hospitalisés}

Dans un arrêt du 13 juin $2001^{53}$, le Conseil d’État a précisé que les dispositions de l'article R. 716-9-1 du Code de la santé publique relatives à l'engagement d'acquitter tous les frais de séjour non pris en charge par l'aide médicale ou l'assurance maladie n'ont pas pour objet et ne peuvent avoir légalement pour effet de placer l'hospitalisé, qui a la qualité d'usager d'un service public administratif, dans une situation contractuelle ${ }^{54}$.

Cette analyse a été confirmée dans un arrêt du Conseil d'État du 27 janvier $2014{ }^{55}$ précisant que la personne hospitalisée dans un établissement public de santé est un usager d'un service public administratif et que le rapport né de cette situation est un rapport de droit public.

\section{c. L'absence de charte " contractuelle » de thèse}

Dans un premier arrêt en date du 20 mars $2000^{56}$, le Conseil d'État était saisi d'un recours contre un arrêté du ministre de l'Éducation nationale publiant une chartetype des thèses conclue entre doctorant, directeur de thèse et structure d'accueil. La haute juridiction administrative a considéré à l'occasion qu'en énonçant que la charte est signée par le directeur de thèse et le doctorant, l'arrêté attaqué n'a pas pour objet et ne pourrait légalement avoir pour effet d'établir une relation contractuelle entre les signataires. L'arrêté ne portait donc pas atteinte à la liberté contractuelle des enseignants-chercheurs ni au caractère réglementaire qui s'attache à la situation du doctorant par rapport au service public de l'enseignement supérieur.

Dans un second arrêt, en date du 21 décembre 2001, le Conseil d'État confirme l'absence de charte «contractuelle» de thèse :

Considérant $[\ldots]$ que par une délibération de son conseil d'administration du 8 mars 1999 l'université Paris VI a approuvé une charte des thèses; que s'il est prévu qu'au moment de son inscription le doctorant «signe» avec le directeur de thèse "la présente charte», une telle indication implique simplement que les intéressés ont pris connaissance de ce document et n'a pas pour objet et ne pourrait d'ailleurs avoir légalement pour effet d'établir une relation de nature contractuelle entre les signataires; qu'eu égard à la circonstance que les usagers du service public de l'enseignement supérieur sont placés à l'égard de ce dernier dans une situation réglementaire, les dispositions de la charte des thèses adoptée par l'université Paris VI, s'appliquent aux doctorants dont les travaux sont en cours à la date de son adoption; qu'au nombre de ces dispositions figurent celles du paragraphe 7 intitulé «Procédures de médiation»; que lesdites procédures, qui ne revêtent qu'un caractère facultatif, habilitent le président à "prendre tous les avis nécessaires afin de résoudre le conflit» survenu entre un doctorant et un directeur de thèse ${ }^{57}$.

51. CE, 5 juillet $2017, \mathrm{n}^{\circ} 399977$, cons. 1 (nous soulignons).

52. CE, 4 décembre $2019, \mathrm{n}^{\circ} 418975$, cons. 4 et 5

53. CE, 13 juin $2001, \mathrm{n}^{\circ} 211613$.

54. Le Conseil d'État a repris cette formule dans un arrêt en date du 3 février 2016 ( $\mathrm{n}^{\circ} 388643$ ) à propos d'une personne bénéficiant pourtant, dans un hôpital, d'un "contrat d'hébergement ".

55. CE, 27 janvier 2014, $\mathrm{n}^{\circ} 359582$.

56. CE, 20 mars $2000, \mathrm{n}^{\circ} 202295$.

57. CE, 21 décembre 2001, $\mathrm{n}^{\circ} 220997$ (nous soulignons). 


\section{L'impossible bénéfice du régime de la collaboration occasionnelle au service}

Un usager d'un service public administratif victime d'un dommage à l'occasion de sa collaboration à ce service public ne pourra pas bénéficier du régime de responsabilité sans faute propre aux dommages subis par les collaborateurs occasionnels du service public. Le Conseil d'État a précisé cette exclusion à l'occasion d'actions formées par un élève d'une école publique victime d'un accident lors d'épreuves sportives (a) et par des éleveurs blessés à l'occasion d'opérations de vaccination de leur bétail (b).

\section{a. L'aide au bon déroulement d'une épreuve sportive ne confère pas à l'usager du service public administratif la qualité de collaborateur occasionnel du service public}

Dans une affaire relative à un accident subi par un élève dans une école publique de la commune de Saint-GermainLangot à l'occasion d'épreuves du brevet sportif scolaire (cet élève avait été frappé au visage par une corde élastique servant à l'épreuve de saut qu'il tenait au moment de l'accident), le Conseil d'État a jugé le 23 juin $1971^{58}$ que la responsabilité de la commune ne pouvait être engagée sur le terrain du défaut d'entretien d'un ouvrage public communal car la corde en cause était tendue entre deux poteaux métalliques non fixés au sol. La responsabilité de la commune ne pouvait davantage être engagée sur le terrain de la faute car le matériel scolaire utilisé n'était pas défectueux. Restait donc à envisager la mise en jeu de la responsabilité de la commune sur le terrain de la collaboration occasionnelle au service public. Ce dernier fondement de responsabilité a été écarté par le Conseil d'État au motif que l'exercice scolaire sportif au cours duquel l'élève a été blessé se déroulait dans le cadre habituel des activités d'enseignement de l'établissement si bien que l'aide fournie pour tenir la corde ne lui a pas conféré la qualité de collaborateur occasionnel du service public (service public qui n'aurait pu en toute hypothèse qu'être un service public de l'État de l'enseignement public du premier degré et non un service public communal).

\section{b. L'aide au bon déroulement d'une vaccination ou d'un prélèvement sanguin anti-brucellose ne confère pas à l'éleveur la qualité de collaborateur occasionnel du service public}

Dans une espèce jugée par le Conseil d'État le 29 avril 1983, un vétérinaire venait vacciner tout le troupeau d'un éleveur contre la brucellose en exécution d'un mandat sanitaire délivré par le préfet de la Nièvre. Un veau fit un écart intempestif au moment de l'opération et la seringue vint piquer l'épaule de l'éleveur lui causant une interruption temporaire de travail d'un mois, deux mois d'incapacité à $50 \%$ et $12 \%$ d'invalidité permanente partielle. L'éleveur a certes obtenu réparation de son préjudice suite à ses blessures mais non sur le terrain de la collaboration occasionnelle au service public, l'usager du service ne pouvant à la fois bénéficier du service - critère de sa qualification d'usager - et d'un régime de réparation sans faute tout aussi bénéfique.

Considérant que M. X... qui, selon les pièces du dossier, assistait passivement à l'opération et, en raison des avantages qu'il en retirait, était un usager du service public de la vaccination vétérinaire, ne saurait être pour ces raisons regardé comme un collaborateur occasionnel de ce service; Considérant en revanche que le vétérinaire, en ne s'assurant pas de la contention de l'animal avant de procéder à l'opération de vaccination et en faisant preuve d'une particulière maladresse malgré l'écart de l'animal, a commis une faute de nature à engager la responsabilité de l'État pour le compte duquel il intervenait; qu'aucune faute ne saurait en revanche être reprochée à M. X... ${ }^{59}$.

Dans une affaire du 9 mars 2010, la cour administrative d'appel de Bordeaux a statué sur le cas d'un vétérinaire qui procédait, en exécution d'un mandat sanitaire délivré par le préfet dans le cadre d'une opération de recherche de la brucellose, à un prélèvement sanguin sur un bovin appartenant à M. X, exploitant agricole. Ce dernier avait alors subi une ruade de l'animal qui l'a blessé au genou. La cour administrative d'appel de Bordeaux applique en la matière les principes dégagés par le Conseil d'État dans son arrêt précité du 29 avril 1983 en des termes quasiment identiques pour écarter toute application du régime jurisprudentiel de la collaboration occasionnelle au service public:

[...] qu'en raison des avantages qu'il en retirait, M.X était un usager du service public de la vaccination vétérinaire; que, dès lors, il ne saurait être regardé comme un collaborateur occasionnel de ce service; que si M. X assurait la contention de son animal lorsque l'accident est survenu, le concours qu'il apportait ainsi au service public, d'ailleurs prévu par les dispositions de l'arrêté du 20 mars 1990 fixant les mesures techniques et administratives relatives à la police sanitaire et à la prophylaxie collective de la brucellose bovine, n'excède pas celui qui peut normalement être demandé à tout propriétaire d'un animal à l'occasion d'un prélèvement sanguin; que, par suite, c'est à tort que le Tribunal administratif de Limoges a jugé que l'État est responsable, sur le fondement du risque, des conséquences dommageables pour $\mathrm{M}$. X des blessures dont il a été victime ${ }^{60}$. 